\title{
Multiple Endocrine Organ Failure due to Amyloidosis
}

\author{
Feyzi GÖKOSMANOĞLU, Çiğdem TURA BAHADIR, Elif KILIÇ KAN, Ayșegül ATMACA \\ Ondokuz Mayis University School of Medicine \\ Department of Endocrinology and Metabolism \\ Samsun, TURKEY
}

\section{OBJECTIVES}

Amyloidosis is characterized by accumulation of amorphous and protein substance in several tissues and organs. Thyroid, adrenal and pituitary insufficiencies may rarely be observed due to accumulation of these substances. Here, we present a case to point out this rare condition.

Table 1: Hormone levels of the patient

\section{CASE REPORT}

Nineteen-year-old male patient admitted with complaints of swelling in the neck, abnormality in thyroid hormone levels and fatigue. He had been diagnosed with familial mediterranian fever at the age of 5 . At 13 years old, he had renal insufficiency due to amyloidosis and had undergone renal transplantation. Physical examination revealed systemic arterial blood pressure of $90 / 60 \mathrm{mmHg}$, pulse rate of 72 beats $/ \mathrm{min}$. Thyroid gland was found to be diffuse palpable (Grade 2), secondary sex characteristics development was defined as stage 2-3. TSH level was increased, FT4 level was decreased, Anti-TPO and Anti-TG were negative. He had primary adrenal insufficiency and hypogonadotrophic hypogonadism. ACTH and $\mathrm{GnRH}$ stimulation tests were in concordance with primary adrenal failure and hypogonadotrophic hypogonadism. Thyroid ultrasonography revealed that right thyroid lobe was $24 \times 23 \times 54 \mathrm{~mm}$ and left thyroid lobe was $17 \times 21 \times 52 \mathrm{~mm}$ in diameters. Isthmus was $5.5 \mathrm{~mm}$ in diameter and there was heterogenic echogenity in thyroid parenchyma. Doppler ultrasonography revealed Grade 1-2 increased thickness in thyroid parenchyma. Thyroid fine needle aspiration biopsy revealed homogenous eosinophylic accumulation which was stained with congo red and methyl violet between thyroid follicullar cells. Pituitary magnetic resonance imaging was normal. In the light of these findings the patient was diagnosed with amyloid goiter, primary adrenal insufficiency and partial pituitary insufficiency. Prednisolone $5 \mathrm{mg} /$ day, levothyroxine $50 \mu \mathrm{g} / \mathrm{day}$ and testosterone enanthate $125 \mathrm{mg}$ every three weeks were administered.

\begin{tabular}{l|l|l|}
\hline & Level & Reference Range \\
\hline TSH & $12.6 \mu \mathrm{IU} / \mathrm{ml}$ & $0.51-4.3 \mu \mathrm{IU} / \mathrm{ml}$ \\
\hline FT4 & $0.78 \mathrm{ng} / \mathrm{dl}$ & $0.93-1.7 \mathrm{ng} / \mathrm{dl}$ \\
\hline ACTH & $172 \mathrm{ng} / \mathrm{dl}$ & $0-46 \mathrm{pg} / \mathrm{ml}$ \\
\hline Cortisole & $2.89 \mu \mathrm{g} / \mathrm{dl}$ & $6.2-19.4 \mu \mathrm{g} / \mathrm{dl}$ \\
\hline FSH & $2.7 \mathrm{mU} / \mathrm{ml}$ & $3.5-12.5 \mathrm{mU} / \mathrm{ml}$ \\
\hline LH & $4 \mathrm{mIU} / \mathrm{ml}$ & $2.4-12.6 \mathrm{mIU} / \mathrm{ml}$ \\
\hline Total Testosteron & $1.74 \mathrm{ng} / \mathrm{ml}$ & $2.8-8 \mathrm{ng} / \mathrm{ml}$ \\
\hline Free Testosteron & $4.1 \mathrm{ng} / \mathrm{ml}$ & $8.6-54 \mathrm{ng} / \mathrm{ml}$ \\
\hline
\end{tabular}

TSH: thyroid - stimulating hormone FT4: Free thyroxine ACTH: Adrenocorticotropic hormone FSH: follicle-stimulating hormone LH: Luteinizing hormone

\section{CONCLUSIONS}

Systemic amyloidosis may be accompanied with amyloid accumulation in thyroid gland as well as adrenal and pituitary insufficiencies. Thus, endocrine evaluation is necessary in these patients. 